\title{
Combined EELS and XAS Analysis of the Relationship between Depth Dependence and Valence in LSMO Thin Films
}

Jim Fitch ${ }^{1}$, Robbyn Trappen ${ }^{2}$, Chih-Yeh Huang ${ }^{2}$, Jinling Zhou ${ }^{2}$, Guerau Cabrera ${ }^{2}$, Shuai Dong ${ }^{3}$, Shalini Kumari $^{2}$, Mikel B. Holcomb ${ }^{2}$, and James M. LeBeau ${ }^{1}$

1. Department of Materials Science and Engineering, North Carolina State University, Raleigh, NC USA

2. Department of Physics and Astronomy, West Virginia University, Morgantown, WV USA

${ }^{3 .}$ Department of Physics, Southeast University, Nanjing, China

Lanthanum Strontium Manganite (LSMO) thin films are of interest due to their colossal magnetoresistive properties, which originates from double exchange related to the Mn 3+ to Mn 4+ charge states [1]. The local magnetic properties of the film are also directly related to the lattice strain at the film/substrate interface, and are tied to the strong lattice-electron interaction related to the $\mathrm{MnO}_{6}$ octahedra in the perovskite structure. The distortion of these octahedra is related to both mechanical strain caused by film/substrate misfit and the radial difference between La and $\mathrm{Sr}$ [2]. While x-ray characterization methods have illuminated oxidation states in these systems, [3] prior x-ray studies lack the spatial resolution of TEM. While the $3^{+}$to $4^{+}$trend as a function of LSMO thin film thickness is well established [1], the relationship between depth dependence and charge state along with accompanying structural effects is not well documented.

In this talk, we will discuss the results of parallel analysis between XAS and EELS on the same films using the same data processing methods for both forms of spectra. To examine the relationship between charge state and depth dependence of ultra-thin $\mathrm{L}_{0.33} \mathrm{Sr}_{0.67} \mathrm{MnO}_{3}$ films, samples of various thicknesses are deposited on STO substrates by pulsed laser deposition. The recorded data is analyzed using the method of least squares fit to $3^{+}$and $4^{+}$reference spectra, collected using $\mathrm{Mn}_{2} \mathrm{O}_{3}$ and $\mathrm{MnO}_{2}$ powders. Figure 1a shows the results of this type of analysis in a representative region of the thin film sample. A map of valence state across the region shown in figure $1 \mathrm{~b}$, shows an increasing average Mn valence from interface to film surface as shown in figure 1c. We will discuss the results of a one-one comparison between XAS and EELS, providing validation of consistency between the two methods.

Regarding lattice strain effects, it has also been shown that these films display an extended out of plane lattice parameter in the first few unit cells near the interface [8]. To further elucidate the magnetic properties of LSMO thin films, strain information was gathered and then processed to provide direct lattice displacements measurements from atomic resolution RevSTEM images [7]. Using accurate and precise revolving STEM measurements, our LSMO thin films are consistent with these prior works. Figure 2 shows that the out of plane strain on both the $\mathrm{A}(\mathrm{La} / \mathrm{Sr})$ and $\mathrm{B}(\mathrm{Mn})$ sites near the interface reaches as high $2 \%$, although, this degree of strain occurs over a narrower region on the A sites, than it does with respect to the B sites. Atom column intensities were then paired with the strain measurements to relate local chemistry to the degree of lattice strain. We will show that using these techniques in conjunction XAS experiments provide a more complete picture of the electronic behavior of these films, as it relates to both structure and chemistry [9]. 
References:

[1] Y. Moritomo, et al., Nature, 380 (1996), pp. 141.

[2] A. Vailionis, et al., Phys. Rev. B, 83 (2011), p. 64101.

[3] C. Aruta, et al., Phys. Rev. B, 73 (2006), p. 235121.

[4] F. Pailloux, et al., Phys. Rev. B, 66 (2002), p. 14417.

[5] L. Samet, et al., Eur. Phys. J. B - Condens. Matter Complex Syst., 34 (2003), pp. 179.

[6] Z. Shouliang, et al., American Mineralogist, 95 (2010), p. 1741.

[7] X. Sang, et al., Ultramicroscopy, 138 (2014), pp. 28..

[8] M. Nord, et al., Appl. Phys. Lett., 106 (2015), p. 041604

[9] This work is supported by the National Science Foundation (DMR-1608656). The authors also acknowledge the Analytical Instrumentation Facility (AIF) at North Carolina State University, which is supported by the State of North Carolina and the National Science Foundation.
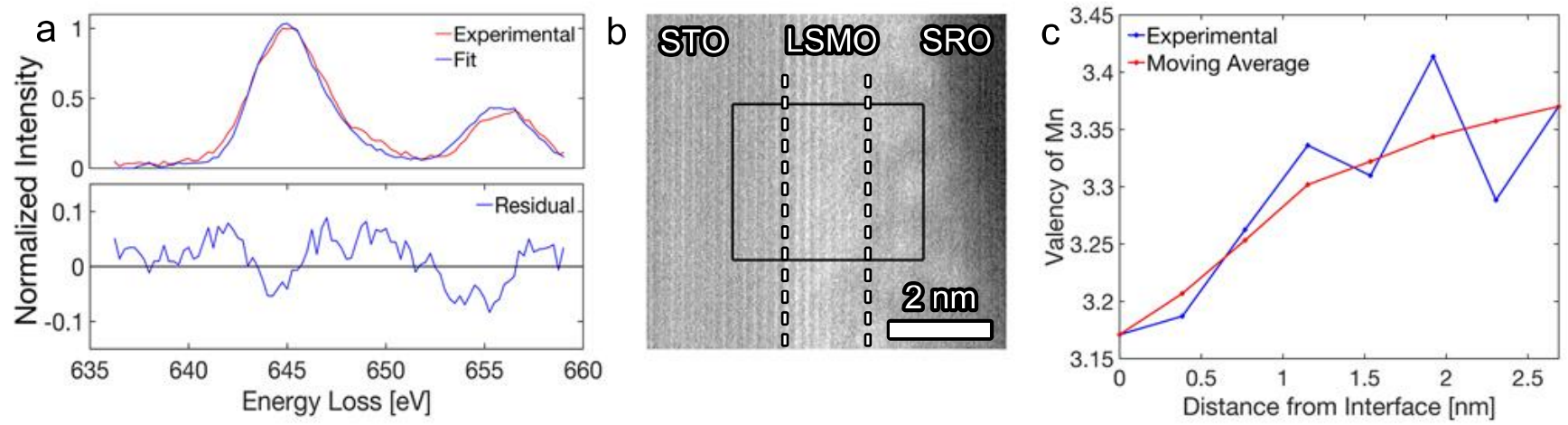

Figure 1. a) EELS spectra plotted with the resultant fit of $\mathrm{Mn}_{3}$ and $\mathrm{L}_{2}$ peaks, and the corresponding residuals. c) STEM image highlighting the region of $\mathrm{Mn}$ valence analysis for c), where the processed region starts at the STO/LSMO interface and ends at the LSMO/SRO interface.
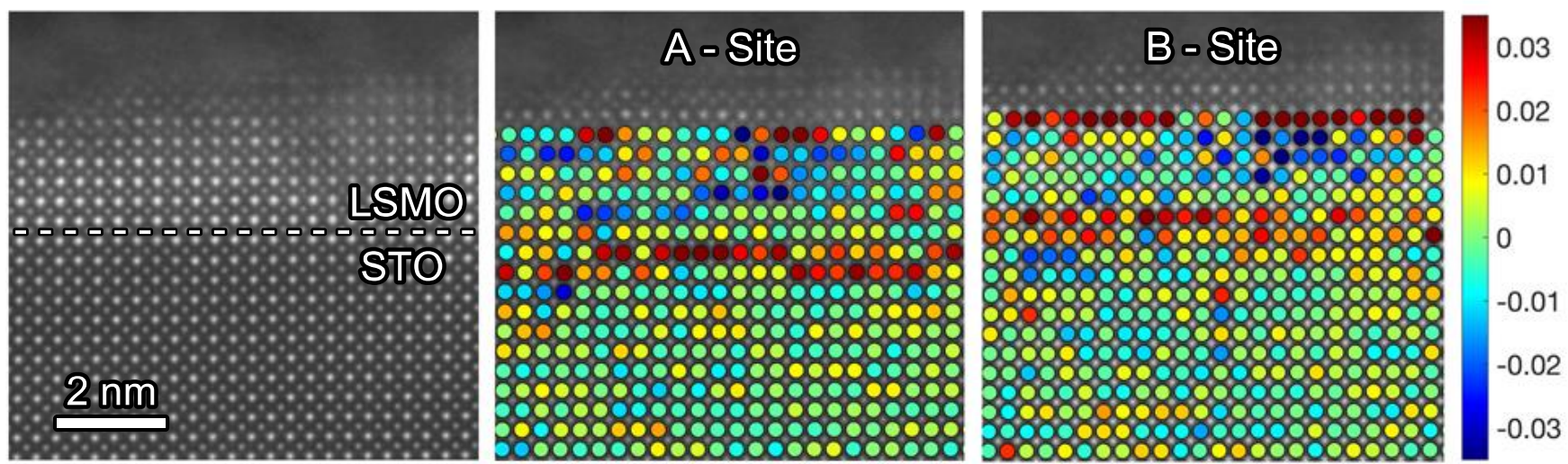

Figure 2. RevSTEM image showing the LSMO/STO interface along with the out-of-plane strain associated with both $\mathrm{A}-$ and $\mathrm{B}-$ sites. 